\title{
Performance Enhancement of Negative Ion Sources for the JT-60U Tokamak
}

\author{
L. R. Grisham*, M. Kuriyama, M. Kawai, T. Itoh, N. Umeda, and JT-60U Team \\ *Princeton Univ. Plasma Phys. Lab., P. O. Box 451, Princeton, N. J. USA 08543 \\ Japan Atomic Energy Research Institute, Naka-Machi, Ibaraki 311-0193 Japan
}

\begin{abstract}
The negative ion based neutral beam system now operating for plasma heating and current drive on the large JT-60U tokamak marked the first application of negative ion source technology to the production of high current, high voltage beams for conversion to neutral atomic beams. This pioneering system has demonstrated the technical feasibility of negative ion based neutral beams for future fusion devices. Because this was a very large advance in the state of the art with respect to all system parameters, the principal physical processes governing the performance of the ion source and accelerator were somewhat different than had been the case with earlier much smaller negative ion sources. We have explored the physical mechanisms limiting the power and pulse length capability of these large sources, and have implemented ameliorating changes to reduce power loading on the accelerator grids and increase the transmitted power fraction.
\end{abstract}

\section{INTRODUCTION}

The JT-60U [1] tokamak in Naka, Japan is one of the largest tokamaks operating in the world today to conduct studies in magnetic confinement nuclear fusion for eventual application to fusion power plants. In the past, many fusion research devices have used beams of energetic neutral hydrogen isotope atoms injected across the confining magnetic field lines to heat the plasma, and also in some cases to drive part of the current circulating in the plasma, which in turn creates the poloidal component of the confining magnetic field. Previous generations [2,3] of neutral beam systems first produced positive ions of the desired hydrogen isotope, electrostatically accelerated the ions, and then converted a portion of the fast ions back to atoms by passage through a gas cell. However, for beam energies above $80 \mathrm{keV}$ for deuterium, this neutralization process becomes rapidly less efficient.

Larger magnetic confinement devices require higher energy hydrogen isotope atomic beams for efficient current drive in the plasma core, and for heating of the central plasma. Negative ions of hydrogen can be converted to neutral atoms by passage through a gas cell with an acceptable efficiency (58-60\%) over a wide range of beam energies. Unfortunately, because the electron affinity of hydrogen is only $0.75 \mathrm{eV}$, it is much more difficult to produce and extract negative hydrogen ions than their positive counterparts.

As the culmination to an extensive development program [4], JT-60U became the first device to use large high-current negative ion sources to produce beams of energetic neutral atoms [5]. Designed with the aim of eventually producing a total of 10 megawatts of neutral beams at $500 \mathrm{keV}$ for 10 sec from two sources on one beamline, this system represented a very large advance in the state of the art in terms of negative hydrogen current, power, and source size. Such a large step is intrinsically challenging, and indeed, during early experiments, the beam transmitted power, pulse length capability, and usable voltage were less than planned. Although these limitations were engendered by a variety of processes, they were all manifest as excessive power deposition upon the accelerator and ground grids of the ion sources due to the interception of the grids by divergent beam particles. The grids were designed with water cooling sufficient to remove steady state heat loads equivalent to about $5 \%$ of the accelerated beam power, but individual grids were being struck by as much as $15 \%$ of the accelerated power. This then limited the beam current and power which could 
be achieved without striking high voltage breakdowns between the grids. Since the optics were of the Pierce weak-focusing variety ubiquitous among ion sources used for fusion neutral beams, the curent limitations translated into limitations upon the usable voltage as well. Several of the principal phenomena limiting the source performance arise from characteristics of large area sources, and thus were less important in their smaller predecessors. This paper addresses measures undertaken to address these phenomena.

\section{BEAM STRIPPING}

One source of the heavy grid interception was excessive stripping of the fragile negative ions while transiting the grid structure. Ions which were stripped to neutrals while still within the accelerator experienced only a subset of the electrostatic lenses, and thus were frozen onto generally divergent trajectories. Since beam ions were neutralized after passing through differing lengths of the accelerating field, some of them had energies lower than the nominal accelerating voltage, producing a lower energy continuum in the beam. The stripping was found to arise from a steeply increasing pressure in the source and accelerator as a function of time, which occurred as a consequence of the long vacuum time constant of the gas feed system. While it was not immediately feasible to change the geometric characteristics that gave rise to this behavior, it was possible to change the gas pulse timing to allow the gas to equilibrate before the arc, which in turn allowed a lower gas throughput to be used. After this improvement, a Doppler shift measurement showed that essentially all of the beam power transmitted to JT-60U was at the full acceleration energy.

\section{SECULAR DEPENDENCE}

After the sharp time dependence of the source and accelerator pressure was corrected, a strong secular dependence still persisted in nearly all of the source parameters, including the arc voltage and current, the extraction current, the extraction grid bias current, and the fraction of co-extracted electrons in the beam. In particular, the arc impedance was declining significantly, which in turn reflected a change in arc characteristics, and consequently a time dependence in the extractable negative ion current density. This led to time-varying divergence in the beam, which increased the average grid interception. It was found that the time required for the arc to equilibrate in negative ion sources of this sort is very long, and that the early operation had been done during the long turn-on transient. This problem was also corrected, so that the source plasma characteristics are equilibrated by the inception of beam extraction. This was accomplished by increasing the time the arc was on prior to beam extraction. The period required for equilibration was a decreasing function of arc and filament power, with 1.5 to 2.0 seconds being sufficient for most conditions, as opposed to the 0.5 second of arc prior to beam extraction which had been used during early operations.

Recently, further control over the secular dependence of the arc has been implemented with a filament control system which allows the programming of eight different values of the filament heating current at different times during the arc and beam pulse. This facilitates stable operation for longer pulses. It also results in lower average filament temperatures than was previously the case, reducing the evaporation of tungsten, and also the incidence of unipolar arcs which erode the filament. It is expected that this will increase filament lifetime, and reduce cesium burial by evaporated tungsten deposited on the source surfaces.

\section{SPATIAL NON-UNIFORMITY}

A strong spatial non-uniformity in the source plasma persisted even in the equilibrated arc, and this in turn led to a non-uniformity in the local negative ion current density extracted from different areas of the source grids. Since the same voltage gradients are applied over the whole area of the grids, local variations in current density result in mismatches between the radially outward force of the beam space charge and the radially inward electrostatic focussing field, and cause position-dependent divergence growth.

A number of diagnostic techniques were used to assess the plasma and negative ion current density non-uniformity. These included a beam-scanning movable calorimeter, the sharpness of reverseaccelerated electron beamlet burn marks on the back of the source, the relative temperatures of the five vertically arrayed sectors of the plasma grid, Langmuir probes, and most usefully, a technique in which the relative magnitudes of the arc currents flowing through each of the eight cathode groups were used as a measure of the relative plasma density in the vicinity of each group. All five of these techniques revealed a consistent view of plasma non-uniformity dominated by a top-down asymmetry, with higher density at the top of the source, declining in the lower third by several tens of percent. This led to a similarlystructured non-uniformity in the accelerated beam 
intensity, which in turn gave rise to increased deposition of power on the accelerator and ground grids, and thus to decreased voltage holding capability.

The most useful of these uniformity assessment techniques was the one we developed using the relative magnitudes of the arc currents flowing through the 8 cathode groups. This data could be easily gathered in a single shot and also had the capability to reveal any time dependence to the spatial nonuniformity. Unlike the Langmuir probes, this technique was not susceptible to contamination by the cesium used in the arc chamber to increase negative ion production.

We developed this assessment technique after realizing that, with the cathodes operating in the space charge limited regime, which had been the customary operating regime for these sources, the current flowing across each cathode sheath was a measure of the local plasma density. Thus, measurements of the arc currents flowing through each of the eight cathode groups yielded a measure of the plasma density in the vicinity of each group of filaments, and thus of the plasma uniformity. These were effectively functioning as reverse Langmuir probes, but without concerns about cesium contamination or extra biasing circuits.

In attempting to understand the mechanism driving the vertical inhomogeneity, all of the source parameters were scanned through their full accessible ranges, and those which could be reversed, such as the grid bias voltage across the plasma grid sheath and the direction of the longitudinal current flowing through the plasma grid to produce an electron filter field in the extraction region, were reversed. The nonuniformity was largely unchanged by any of these variations, and was also little altered by the presence or absence of cesium within the chamber. This leads to the supposition that it is probably driven at least in part by the one parameter that could not be reversed: the direction of arc current flow through the filaments and into the plasma. This gives rise to a continuous magnetic field flowing the length of the source. Unlike the magnetic field produced by the filament heating current, the magnetic field from the arc is not self-canceling. The potential for this causing appreciable non-uniformity is greater for sources with long extraction areas, such as this one (each source weighs 6.2 metric tons) than for its smaller predecessors.

A number of techniques were employed to reduce or compensate for the spatial inhomogeneity. Series resistors were added to the arc circuits for each of the eight filament groups. These resistors were independently adjustable to values between 25 and 150 milliohms, with the lowest values being installed in the regions exhibiting the lowest plasma densities. This produced some success, with decreases of the nonuniformity by a factor of two, but this is not a perfect solution because the required resistor balance tends to vary somewhat with arc and filament power and pulse length.

In order to reduce the power striking the grids due to edge effects, $19 \%$ of the plasma grid extraction area was masked, with equal amounts covered at the top and bottom of the source.

The heating current flowing through the filament cathodes was also varied to alter the operating regime of the sources, and to gain some control over the arc impedance. There are two potential disadvantages to operating the cathodes in the space charge limited mode, as had been the traditional case for these sources. One is that, as mentioned earlier, the current flow across the cathode sheath is proportional to the local plasma density. Thus, more primary ionizing electrons will be accelerated across the cathode sheaths located in high density regions, and less in low density regions. The resulting uneven distribution of ionizing electrons will probably tend to reinforce the intrinsic non-uniformity in the plasma distribution.

The second potential disadvantage to space charge limited cathode operation is that the discharge conditions determine the arc impedance, with the source operator having little independent control over the voltage drop across the cathode sheath, which determines the acceleration energy of the primary electrons. In any given set of discharge conditions, there is presumably some primary electron voltage which is most suitable for optimizing the extractable negative ion current. The freedom to tune for this optimum is partially constrained under space charge limited operation, since the voltage is strongly linked to the arc power.

We experimented with modest reductions in the cathode filament heating power to move the operating regime partly into the emission limited regime, where the current flowing across the sheath is determined by the emission from the thermionic filament, rather than by the space charge limit of the plasma sheath. Under these conditions, the acceleration energy of the primary ionizing electrons is partly controllable by the filament heating current .

Under some conditions, operation of the cathode filaments partly into the emission limited regime produced some increase in the beam power accelerated 
through the grids to the beam calorimeter, without increasing the power incident upon the accelerator and ground grids of the beam accelerator assembly. It is unclear at this time how much of this improvement was due to optimizing extractable negative ion current, and how much might have been due to subtle plasma uniformity improvements. Figure 1 shows the arc voltage as a function of filament heating voltage. In the space charge limited regime, the arc voltage is nearly independent of the filament heating voltage (and therefore filament heating power). In the emission limited regime, the arc voltage changes with the filament heating voltage and power. Figure 2 shows the power transmitted to the calorimeter and the power striking the grids as the discharge is moved from the space charge limited regime of cathode sheath operation towards emission limited operation.

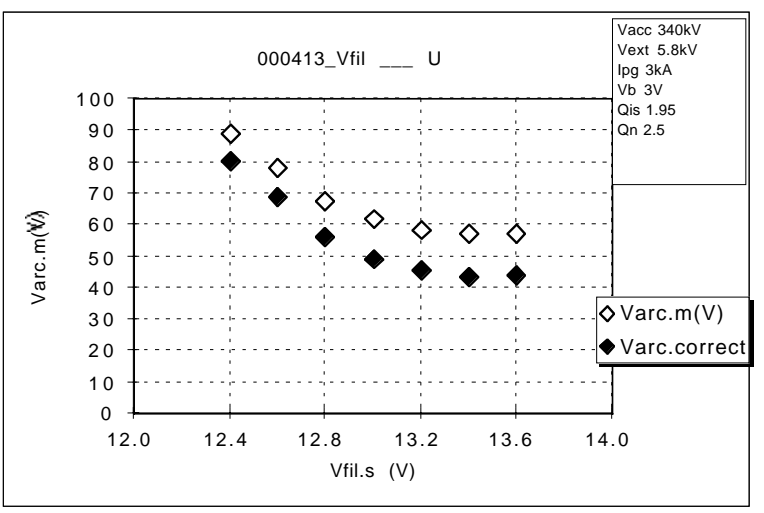

FIGURE 1. The arc enters the emission-limited regime, where the discharge voltage changes, at a filament heating voltage near 12.8 volts. The arc power is $120 \mathrm{~kW}$ throughout the scan.

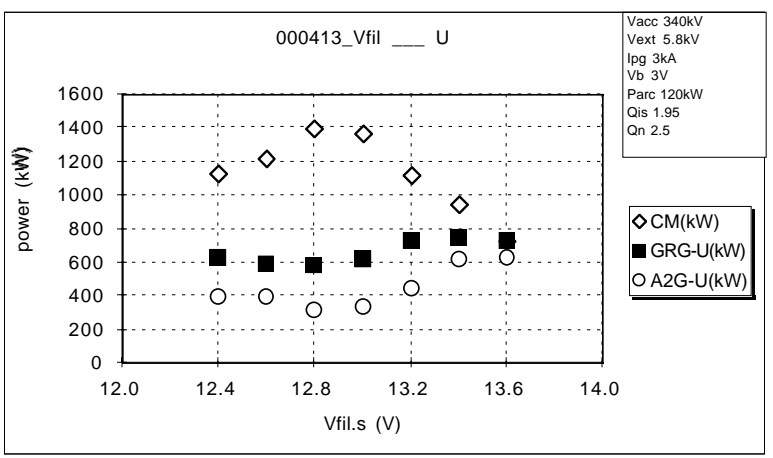

FIGURE 2. Under these arc conditions, some improvement is observed in the form of greater transmitted beam power and reduced grid interception when the discharge is not fully into the space charge limited regime. Diamonds show the power transmitted to the calorimeter, dark squares the power striking the ground grid, and circles the power hitting the second accelerator grid.

\section{CONCLUSION}

This study clarified the role of various physical processes which are important in large cesiated negative ion sources, and it thereby contributed to improved negative ion beam performance on the JT$60 \mathrm{U}$ tokamak. The acceleration efficiency of deuterium has increased from $55 \%$ before these improvements to as high as $72 \%$ afterwards, and to as much as $80 \%$ in the rarely run hydrogen beams. The fraction of the beam power intercepting the accelerator and ground grids has declined by amounts typically in the range of $30-40 \%$. The maximum injected deuterium neutral beam power achieved so far as a result of these improvements is 5.2 megawatts at 350 $\mathrm{keV}$ for 0.77 second [5], and the maximum pulse length is 2.0 seconds at 4.0 megawatts and $360 \mathrm{keV}$.

\section{REFERENCES}

1. M. Kuriyama et. a1., Journal of Nuc. Sci. and Tech., 35, 739-742 (1998).

2. Y. Okumura et. al., Rev. Sci. Instrum. 67, 1018-1022 (1996).

3. T. Oikawa et. al., Proc. $17^{\text {th }}$ International Conf. Of Fusion Energy, Yokohama Oct. 1998.

4. T. Oikawa at. al., to appear in Proc. $18^{\text {th }}$ International Conf. On Fusion Energy, Sorrento Oct. 2000.

5. M. Kuriyama et. al., Rev. Sci.Instrum. 71, no. 2, pt II, 751-755 (2000). 